\title{
Study on Radial Stiffness of Contact Pair of Logarithmic Modified Tapered Roller Bearing
}

\author{
Shimin $\mathrm{LUO}^{1}$, and Junyi WANG \\ School of Mechatronics \& Vehicle Engineering, East China Jiaotong University, \\ Jiangxi, Nanchang 330013, China
}

\begin{abstract}
Based on Hertz elastic contact theory, elastohydrodynamic lubrication theory, the equivalent radial stiffness model of roller raceway contact pair of logarithmic modified tapered roller bearing is derived under the consideration of oil film stiffness. The calculation results show that the equivalent radial contact stiffness increases with the increase of contact load, increases linearly with the increase of effective contact length of roller, and changes little with the increase of modification $\mathrm{fl}$. When the radial load is small, the oil film has a great influence on the equivalent radial stiffness, which decreases gradually with the increase of the radial load.
\end{abstract}

Keywords. Tapered roller bearing, logarithmic modification, radial stiffness, elastohydrodynamic lubrication

\section{Introduction}

The axle box bearing of high-speed railway is tapered roller bearing, and its generatrix is linear roller, which has "edge effect". The logarithmic modified roller can reduce the "edge effect", improve the contact stress distribution between the roller and raceway, and improve the eccentric load effect of the roller, which have been widely studied [1]. The contact deformation and radial contact stiffness of bearing contact pairs are the basis of bearing load distribution and dynamic characteristics analysis. Fu Yuanning has analyzed the influence law of elastic deformation and contact stress of tapered roller bearing based on Hertz contact theory and finite element method [2]. Houpert has deduced the calculation formula of contact deformation between tapered roller and raceway based on Hertz's contact deformation formula [3]. Wang Ailin have deduced the calculation formula of contact deformation and contact stress distribution of tapered roller bearings by using the influence coefficient method [4]. The contact stiffness of cylindrical roller bearings have been analyzed by SHRINIWAS based on elastohydrodynamic lubrication theory and line contact theory [5]. Wu Hao has derived the radial contact stiffness of the tapered roller bearing under the maximum load[6], but did not consider the equivalent stiffness of the inner and outer ring stiffness in series. Liu Guanghui used the finite element contact analysis method to analyze the stiffness of cylindrical roller bearing [7]. Wu Zhenghai has established a full dynamic model of

${ }^{1}$ Luo Shimin, School of Mechatronics \& Vehicle Engineering, East China Jiaotong University, 808 Shuanggang East Street, Nanchang, Jiangxi, The CHINA; E-mail: 102136900@qq.com . 
tapered roller bearing cage with equivalent stiffness and damping of lubricating oil film [8]. The above roller raceway contact deformation formula is based on the point contact formula, which need to be solved by numerical method and transcendental equation. The efficiency of dynamic characteristic analysis is low. The corresponding stiffness calculation model of tapered roller bearing needs to be simplified.

In this paper, the contact deformation formula of the logarithmic tapered rollers is derived by Hertz elastic contact theory. According to the elastohydrodynamic lubrication theory (EHL), the oil film thickness and stiffness formula are obtained. The radial equivalent stiffness model of roller raceway contact pair of logarithmic modified tapered roller bearing is established, which provides a theoretical basis for dynamic analysis of modified tapered roller bearings.

\section{Contact Deformation}

According to the taper roller slicing processing method [6,9], the taper is cut into $\mathrm{n}$ rectangular elements along the generatrix direction, as shown in Figure $1 . D_{\min }$ is the small end diameter of roller, $D_{\max }$ is the big end diameter of roller, $\beta$ is the half cone angle of roller, $l_{e}$ is the effective contact length of roller. When the distance from the roller top angle is $l_{i}$, the diameter of the section perpendicular to the roller center line is

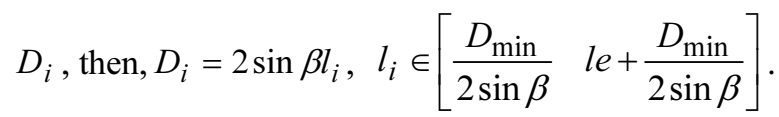

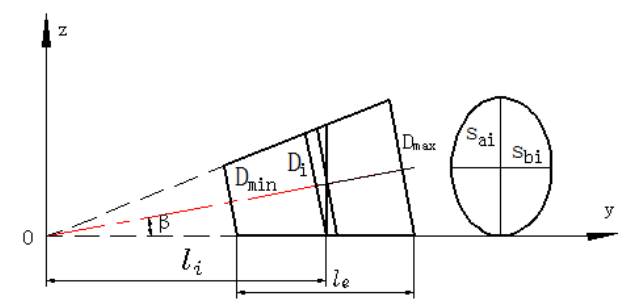

Figure 1. The geometrical relationship of tapered roller

The $S_{a i}$ and $S_{b i}$ is the long and short axes of the ellipse at the section, the curvature radius of tapered roller and inner raceway at the slice is as follows.

$$
R_{i}=\frac{\left(\frac{s_{b i}}{2}\right)^{2}}{\frac{s_{a i}}{2}}=\frac{1}{2} \frac{1+\tan \beta \tan 2 \beta}{\cos \beta+\sin \beta \tan 2 \beta} D_{i}=A_{1} D_{i}, \quad R_{1 i}=\frac{1}{2} \frac{1+\tan \alpha \tan 2 \alpha}{\cos \alpha+\sin \alpha \tan 2 \alpha} D_{1 i}=A_{2} D_{1 i}
$$

The curvature radius of the outer raceway at the slice is as follows.

$$
R_{2 i}=\frac{1}{2} \frac{1+\tan (\alpha+2 \beta) \tan 2(\alpha+2 \beta)}{\cos (\alpha+2 \beta)+\sin (\alpha+2 \beta) \tan 2(\alpha+2 \beta)} D_{2 i}=A_{3} D_{2 i}
$$


Where, $D_{1 i}$ and $D_{2 i}$ are the section diameters of the inner and outer raceways parallel to the raceway end face at the contact point. In this paper, the lower subscript 1 indicates the inner ring, the lower subscript 2 indicates the outer ring. According to the basic formula of plane contact problem [10], the half width and contact deformation of convex -convex contact are shown in Eq. (1).

$$
b=1.59 \sqrt{\frac{Q}{l_{e}} \frac{R_{a} R_{b}}{R_{a}+R_{b}} \frac{1-v^{2}}{E}}, \quad \delta=\frac{2}{\pi} \frac{Q}{l_{e}} \frac{1-v^{2}}{E}\left(\ln \frac{4 R_{a} R_{b}}{b^{2}}+0.834\right)
$$

The half width and contact deformation of convex- concave contact are shown in Eq. (2).

$$
b=1.59 \sqrt{\frac{Q}{l_{e}} \frac{R_{a} R_{b}}{R_{a}-R_{b}} \frac{1-v^{2}}{E}} \quad, \quad \delta=\frac{2 Q}{l_{e}} \frac{1-v^{2}}{E}(1-\ln b)
$$

Where, $v$ is Poisson's ratio, $\mathrm{E}$ is the elastic modulus, $R_{a}, R_{b}$ are the curvature radius of two contact bodies, Q is the normal load. The contact deformation between the roller and the inner or outer raceways can be obtained by substituting $R_{i}, R_{1 i}$ and $R_{2 i}$ into the Eqs. (1) and (2), as shown in Eqs. (3) and (4).

$$
\begin{aligned}
& \delta_{1 i}=\frac{2 Q}{\pi l_{e} E^{\prime}}\left[\ln l_{i}+\ln \frac{\left(A_{1}+A_{2}\right) l_{e} E^{\prime} \sin \beta}{Q}+1.986\right] \\
& \delta_{2 i}=\frac{Q}{l_{e} E^{\prime}}\left[-\ln l_{i}-\ln \frac{Q A_{1} A_{3} \sin \beta}{l_{e} E^{\prime}\left(A_{3}-A_{1}\right)}+0.38\right]
\end{aligned}
$$

The logarithmic modification roller can eliminate the "edge effect", and the logarithmic modification formula is shown in Eq. (5) [9].

$$
\Delta=f_{1} \ln \left[1-f_{2}\left(2 x / l_{e}\right)^{2}\right]^{-1},|x| \leq 0.5 l_{e}
$$

Where, $f_{1}, f_{2}$ are the logarithmic modification parameter, the unit of $f_{1}$ is um, $f_{2}$ is dimensionless. The contact deformation of logarithmic modified roller and raceway are $\delta_{1 i}^{\prime}=\delta_{1 i}+\Delta$ and $\delta_{2 i}^{\prime}=\delta_{2 i}+\Delta$.

The stress concentration at both ends of the roller length of logarithmic modified tapered roller bearing is significantly reduced, and the stress in the middle part is larger $[11,12]$. Therefore, the deformation of the inner and outer rings at the middle of roller length $\left(l_{i}=\frac{l_{e}}{2}+\frac{D_{\min }}{2 \sin \beta}\right)$ are shown in Eq. (6). 


$$
\left\{\begin{array}{c}
\delta_{1}=m_{1} Q \ln Q+n_{1} Q \\
\delta_{2}=m_{2} Q^{\prime} \ln Q^{\prime}+n_{2} Q^{\prime}
\end{array}\right.
$$

Where, $\mathrm{Q}$ is normal maximum load of inner $\operatorname{ring}, Q=\frac{4.08 F_{r}}{Z \cos \alpha}, Q^{\prime}$ is normal maximum load of outer ring, $Q^{\prime}=\frac{4.08 F_{r}}{Z \cos (\alpha+2 \beta)} \cdot m_{1}=-\frac{2}{\pi_{e} E^{\prime}}, m_{2}=-\frac{1}{l_{e} E^{\prime}}$,

$$
n_{1}=\frac{2}{\pi l_{e} E^{\prime}}\left[\ln \frac{\left(l_{e} \sin \beta+D_{\min }\right)\left(A_{1}+A_{2}\right) l_{e} E^{\prime}}{2}+1.986\right], \quad n_{2}=\frac{1}{l_{e} E^{\prime}}\left[-\ln \frac{\left(l_{e} \sin \beta+D_{\min }\right) A_{1} A_{3}}{2 l_{e} E^{\prime}\left(A_{3}-A_{1}\right)}+0.38\right]
$$

\section{Calculation of Oil Film Thickness of Logarithmic Modified Raceway Contact Pair}

According to the EHL characteristics of the contact pair between the modified roller and the raceway, Dowson have gave the formula of the center oil film thickness of the modified roller $[13,14]$

$$
h_{c}=2.69 \frac{\alpha^{0.53}\left(\eta_{0} u\right)^{0.67} R_{e}^{0.454}}{E^{\prime 0.073} Q^{0.067}}\left(1-0.61 e^{-0.73 k_{e r}}\right)=A_{C} Q^{-0.067}
$$

Where, $\alpha$ is the viscosity compression coefficient, $\eta_{0}$ is the viscosity of lubricating oil, $\mathrm{u}$ is the suction speed, $k_{e}$ is the contact ellipse ratio, $k_{e}=1.0339\left(R_{v} / R_{e}\right)^{0.636}, R_{\mathrm{v}}$ is the equivalent radius of curvature perpendicular to the suction velocity direction, $A_{c}$ is the load film thickness factor.

$$
R_{\mathrm{v}}=\left|\frac{\left(1+\dot{z}^{2}\right)^{3 / 2}}{\ddot{z}}\right|, \quad A_{c}=2.69 \frac{\alpha^{0.53}\left(\eta_{0} u\right)^{0.67} R_{e}^{0.454}}{E^{\prime 0.073}}\left(1-0.61 e^{-0.73 k_{e r}}\right)
$$

$$
\text { Where, } \dot{z}=\frac{8 f_{1} f_{2} x}{l_{e}^{2}\left[1-f_{2}\left(2 x / l_{e}\right)^{2}\right]}, \quad \ddot{z}=\frac{8 f_{1} f_{2}}{l_{e}^{2}}\left[\frac{1}{1-f_{2}\left(2 x / l_{e}\right)^{2}}+\frac{8 f_{2} x^{2}}{l_{e}^{2}\left[1-f_{2}\left(2 x / l_{e}\right)^{2}\right]}\right]^{2}
$$

The curvature radius at the center of contact pair $(\mathrm{x}=0)$ of logarithmic modified roller raceway is $R_{\mathrm{v}}=l_{e}^{2} / 8 f_{1} f_{2}$.

\section{Equivalent Radial Stiffness of Logarithmic Modified Tapered Roller Bearing}

According to the mechanical characteristics of contact pairs by EHL, the contact area is divided into lubricant inlet area, Hertz contact area and outlet area, as shown in Figure 2. 


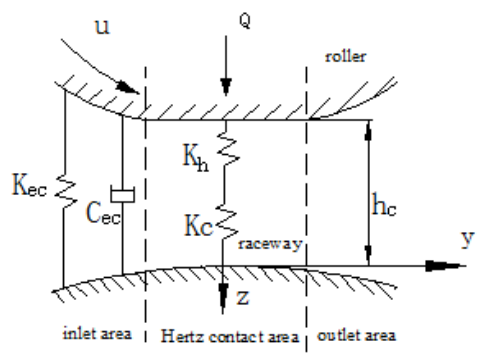

Figure 2. Contact model of contact pair

According to the definition of contact pair stiffness, Hertz contact stiffness of inner and outer raceway contact pairs can be obtained by Eq. (6), which is shown in Eq. (8).

$$
\left\{\begin{array}{c}
K_{h 1}=\frac{\partial Q}{\partial \delta}=\frac{1}{n_{1}+m_{1}+m_{1} \ln Q} \\
K_{h 2}=\frac{\partial Q}{\partial \delta}=\frac{1}{n_{2}+m_{2}+m_{2} \ln Q^{\prime}}
\end{array}\right.
$$
(9).

The oil film stiffness of the contact area can be obtained by Eq. (7), as shown in Eq.

$$
K_{f}=\frac{\partial Q}{\partial h_{c}}=\frac{A_{C}{ }^{14.92537} h_{c}{ }^{-15.92537}}{-0.067}
$$

Since the contact stiffness and oil film stiffness of the contact area are in series, the equivalent stiffness of the roller raceway contact area under lubrication, and the oil film stiffness in the entrance area of logarithmic modified roller and raceway contact pair is as follows.

$$
K_{E_{1}}=\frac{K_{h 1} K_{f}}{K_{h 1}+K_{f}}, \quad K_{E_{2}}=\frac{K_{h 2} K_{f}}{K_{h 2}+K_{f}}, \quad K_{e f}=\frac{8 \eta_{0} u R_{e} a}{h_{c}^{2}\left(K_{f} / K_{h 1}+1\right)}
$$

Where, $a$ is the half length of contact between roller and raceway under dry friction contact.

According to the series parallel relationship of the contact area stiffness of the inner and outer raceways (Figure 2), the equivalent stiffness of the inner and outer raceway contact pairs of the logarithmic modified tapered roller bearing under lubrication can be obtained as $K_{1}=K_{e f}+K_{E 1}$, and $K_{2}=K_{e f}+K_{E 2}$.

The contact stiffness of the inner and outer raceways is in series, so the equivalent stiffness of the bearing contact pair is $K=K_{1} K_{2} /\left(K_{1}+K_{2}\right)$.

For tapered roller bearings with linear contact [7], the load displacement relationship is as follows. $Q=K \delta^{n}, n=1 / 0.9$ 


\section{Calculation Examples and Analysis}

The calculation take the logarithmic modified roller of $35226 \times 2$ double row tapered roller bearing as an example, the effective length of roller is $40 \mathrm{~mm}, \beta=0.806^{0}$, the contact angle of inner ring is $\alpha=7.75^{\circ}, \mathrm{D}_{\min }=22.8 \mathrm{~mm} \mathrm{f1}=4.40 \mu \mathrm{m}, \mathrm{f} 2=0.997$, viscosity compression coefficient is $2.1 \times 10^{-8} \mathrm{~m}^{2} / \mathrm{N}, \eta_{0}=0.02 \mathrm{N.s} / \mathrm{m}^{2}$, $\mathrm{u}=6.8 \mathrm{~m} / \mathrm{s}, \mathrm{E}=2.06 \mathrm{GPa}, \mathrm{v}=0.3, \mathrm{Q}=3.5 \mathrm{kN}$, The radial displacement and radial equivalent stiffness of contact pair calculated by finite element statics analysis are shown in Table 1. The results show that the maximum error between the finite element value and the equivalent stiffness calculation value is about $18 \%$ which verifies the correctness of the equivalent stiffness calculation.

Table 1. Compare of contact radial displacement between finite-element and equivalent stiffness calculation

\begin{tabular}{ccccc}
\hline $\mathbf{Q}(\mathbf{N}))$ & $\begin{array}{c}\text { Effective length } \\
(\mathbf{m m})\end{array}$ & $\begin{array}{c}\text { Finite element } \\
\text { value }(\mathbf{m})\end{array}$ & $\begin{array}{c}\text { Calculated value of } \\
\text { stiffness }(\mathbf{m})\end{array}$ & Error \\
\hline 3500 & 45 & $3.30 \mathrm{E}-04$ & $2.73 \mathrm{E}-04$ & $16.8 \%$ \\
2000 & 45 & $2.21 \mathrm{E}-04$ & $1.86 \mathrm{E}-04$ & $14.6 \%$ \\
3500 & 30 & $4.25 \mathrm{E}-04$ & $3.59 \mathrm{E}-04$ & $14.8 \%$ \\
3500 & 50 & $3.14 \mathrm{E}-04$ & $2.54 \mathrm{E}-04$ & $18.1 \%$ \\
\hline
\end{tabular}

The results of Hertz contact stiffness and equivalent stiffness of tapered roller raceway contact pair are shown in Figure 3-5.

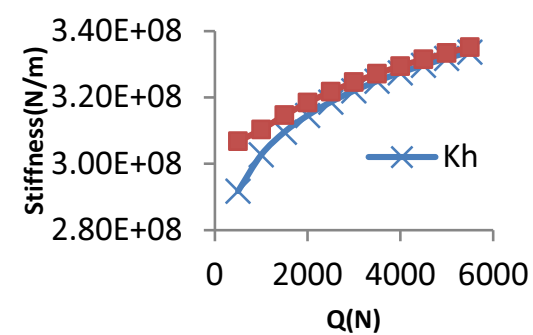

Figure 3. The contact stiffness of the modified roller raceway varies with the load

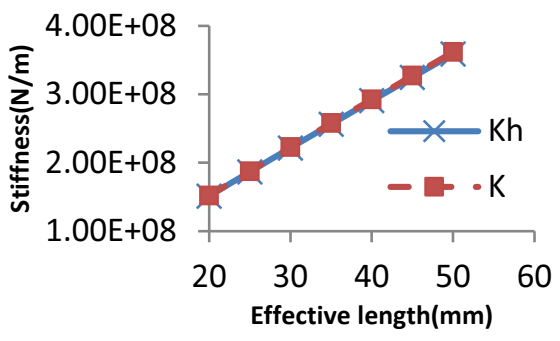

Figure 4. The contact stiffness of the modified roller raceway varies with the 1 the effective length of the roller

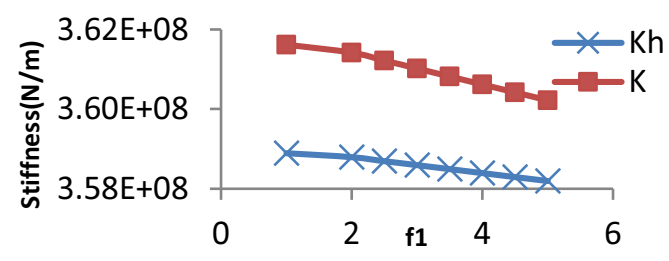

Figure 5. The contact stiffness of the modified roller raceway varies with the modification parameter $f_{1}$

It can be seen that the contact stiffness of logarithmic modified roller raceway increases with the increase of contact load. Under lubrication condition, the equivalent contact stiffness of contact pair is slightly lower than Hertz contact stiffness, and the difference decreases with the increase of load. Under light load, the oil film stiffness has a great influence on the equivalent stiffness, which is due to the decrease of oil film thickness and the increase of oil film stiffness due to the increase of radial load. The contact stiffness increases linearly with the increase of effective contact length, and the 
equivalent contact stiffness is almost equal to Hertz contact stiffness. As the effective contact length increases, the contact surface increases, the contact deformation of roller raceway decreases, so the stiffness increases, and the effective length has no effect on the oil film stiffness. With the increase of modification F1, the contact stiffness decreases in a curve, but the change is small, which can be ignored. Due to the increase of F1, the equivalent radius of curvature in the direction perpendicular to the suction velocity decreases, the oil film thickness increases, and the oil film stiffness decreases.

\section{Conclusion}

Based on Hertz elastic contact theory, the contact deformation of roller raceway contact pair of logarithmic modified tapered roller bearing is deduced. Based on EHL theory and stiffness calculation method, the equivalent stiffness model of roller raceway contact pair of logarithmic modified tapered roller bearing is established. The results show that the equivalent contact stiffness increases with the increase of contact load, increases linearly with the increase of effective contact length of roller, and almost unchanged with the increase of modification F1.

\section{Acknowledgement}

In this paper, the research was sponsored by Jiangxi department project (Project No.GJJ190322and .GJJ180291).

\section{References}

[1] Wei YG. The research on the crowned roller of cylindrical roller bearing. LUBRICATION ENGINEERING,2003,(5):16-18.

[2] Fu YN, Yao TQ, Huang YC. Analysis of contact characteristic of hollow tapered holler bearing. Mechanical drive, 2015(1):114-117.

[3] Houpert L. An engineering approach to non-hertzian contact elasticity - part II. Journal of Tribology -Transactions of The ASME,2001,123( 3) : 589-594.

[4] Wang AL,Wang QJ, Wang JG. Contact of tapered roller with logarithmic profile. Mechanical Science and Technology for Aerospace Engineering,2012,31(5):836-841.

[5] SHRINIWAS PC,MIHIR S.On the dynamics of lubri-cated cylindrical roller bearings,part I: evaluation of stiffness and damping characteristics. Tribology Transactions,2013,56(6): 1087-1096.

[6] Wu H. Study on the calculation method of radial stiffness of tapered roller bearing. Lubrication and sealing,2008,3(7):39-43.

[7] Liu GH, Hong J, Sun YH, el,at. Nonlinear stiffness analysis of cylindrical roller bearing considering the variation of bearing roller positions. China Mechanical Engineering ,2019,6:1-9.

[8] Wu ZH, Xu YQ, Dynamic stability of cages in high-speed tapered roller bearings with grease lubrication. Journal of Vibration and Shock, 2019,38(10):49-57.

[9] Wang C. Contact analysis between roller and raceway of tapered roller bearing in high speed railway. Journal of Beijing Jiaotong University,2017,41(4):91-97.

[10] Chen Q, Lan J, Hua L. Contact stress numerical calcution and convex optimization of tapered roller bearing. Journal of Wunhan university of technology,2016,38(3):91-97.

[11]Wei YG. Symmetrical modification of rolling element of high-speed train axlebox tapered roller bearing. Journal of Dalian Jiaotong University, 2016,37(3):44-47

[12] Wang AL. Contact analysis of logarithmic modified tapered roller. Mechanical science and technology, 2012,31(5):836-8411.

[13] Dowson D. Film Thickness for Different Regimes of Fluid Film Lubrication. NASA, 1983.

[14] Tang HW. Analysis of thermal elastohydrodynamic lubrication of non-Newtonian fluid contact under the condition of large slip-to-roll ratio. LUBRICATION ENGINEERING, 2019,44(9):31-36. 